\title{
STELLAR PHOTOMETRIC OBSERVATIONS AT Ha THROUGH A NARROW-BAND INTERFERENCE FILTER
}

\author{
HUGH E. BUTLER \\ Royal Observatory, Edinburgh, United Kingdom
}

\begin{abstract}
The paper describes the results of stellar photoelectric observations made with a small telescope and a $3 \frac{1}{2} \AA$ wide filter centred at $\mathrm{H} \alpha$.

The method proved to be a sensitive one for detecting change in the spectrum but did not allow differentiation between changes in the $\mathrm{H} \alpha$ profile and wavelength changes due only to the Doppler effect. It was possible, however, to recognise and qualitatively to eliminate the Doppler changes due to the Earth's Annual Motion.

Of ten Be stars examined three, although in strong emission, showed little change over the periods of observation, the longest of which was 9 months. The other seven showed large and random changes.

Warnings are given about the difficulty of tuning such a filter exactly to $\mathrm{H} \alpha$ and the need to monitor accurately the filter for long term changes in passband and throughput.
\end{abstract}

\section{Introduction}

In general, this was an exploratory programme designed to study stellar $\mathrm{H} \alpha$ variations using a $3.5 \AA$ wide filter, a photoelectric photometer and a small telescope: observations were made in Edinburgh between August 1972 and September 1973.

In particular, the work covered:

(a) assessing the particular instrumental set-up;

(b) determining whether rotation periods could be detected in late type stars on the assumption that $\mathrm{H} \alpha$ emission from limited areas of a star's surface would modulate the observed intensity of the $\mathrm{H} \alpha$ line in phase with the rotation period;

(c) studying variation characteristics of $\mathrm{Be}$ stars at $\mathrm{H} \alpha$;

(d) studying a small number of regular variables at $\mathrm{H} \alpha$.

This short paper is mainly concerned with (a) and (c); reference will be made to (b) and (d) but it is intended to describe these in more detail elsewhere.

\section{The Observing Equipment}

A 12-in. telescope was built especially for this work. It was a short Cassegrain instrument with a final beam of focal ratio of 15 . The photometer used a $2^{\prime}$ aperture, a filter slide thermostatted to about $\pm 1^{\circ} \mathrm{C}$, a cooled EMI photomultiplier with an S-20 cathode, photon counting electronics and print out. Emphasis was laid on ease of operation for one observer for long periods in complete darkness.

Two filters only were used:

(a) a Baird interference filter, $3.5 \AA$ halfwidth at half height;

(b) a similar filter $60 \AA$ wide, always used in conjunction with a $10 \%$ transmitting neutral density filter.

A single parameter was derived from the observations, this being the ratio $(R)$ of starlight through the two filters, due allowance having been made for dark and sky 
counts. The purpose of the neutral density filter was to make the counts through the two filters of the same order of magnitude so as completely to avoid any possible trouble due to non-linearity of counting. In practice the values of $R$ ranged from 0.67 (for strong emission) to $\mathbf{2 . 7 0}$ (for broad and strong absorption).

It will be realised that while accurate centring of the passband of the broad filter is unimportant, the reverse is true for the narrow filter. This latter was set by tilt and temperature to be as close as possible to $6562.8 \AA$. Guaranteeing close correspondence proved difficult with the equipment to hand, but it was felt at the time that this correspondence was better than $0.5 \AA$, a value which was considered acceptable compared with a filter half width of $3.5 \AA$. Once the filter was installed and set in the equipment every effort was made to keep it undisturbed. In fact, except for some experiments involving changes in tilt which the equipment allows, the filter was not moved, cleaned or even examined for 11 months. Very soon after, however, a change became apparent and the series had to be abandoned.

\section{The Observations}

The observing list included 43 stars of all spectral types and all brighter than magnitude 5.0.

Conventional comparison stars were not used but two circumpolar stars (see Table I) were chosen for intensive observation with a view to monitoring any changes in the equipment and to give long runs for statistical purposes.

TABLE I

Circumpolar comparison stars

\begin{tabular}{llllll}
\hline Star & $\begin{array}{l}\text { Spectral } \\
\text { type }\end{array}$ & Mag. & Dec. & $\begin{array}{l}\text { Ecliptic } \\
\text { lat. }\end{array}$ & $\begin{array}{l}\text { Nights } \\
\text { observed }\end{array}$ \\
\hline HR3751 & K3 III & 4.44 & $81^{\circ}$ & $60^{\circ}$ & 80 \\
$\begin{array}{l}\text { HR7750 } \\
\text { ( } \text { Cep) }\end{array}$ & B9 III & 4.38 & $78^{\circ}$ & $75^{\circ}$ & 58 \\
\hline
\end{tabular}

Figure 1 shows observed values of $R$ for the 43 stars, the individual ranges of variation being shown as vertical bars. The period over which the observations for any star extended varied considerably depending, as usual, on declination and the relation between right ascension and the sidereal time of the short summer nights. Cases of very few observations are noted in the figure.

Figure 2 plots, for four stars (including the two detailed above), the observed ratios $R$ against date. The smooth curves are drawn by eye.

Data are plotted for ten known or suspected e-type stars in Figures 3 and 4, the curves, again, being drawn by eye.

In addition, various night-long runs were made on stars of known, short period variability. Figure 5 shows the results of an 8 hour session on $\beta$ Cephei. 


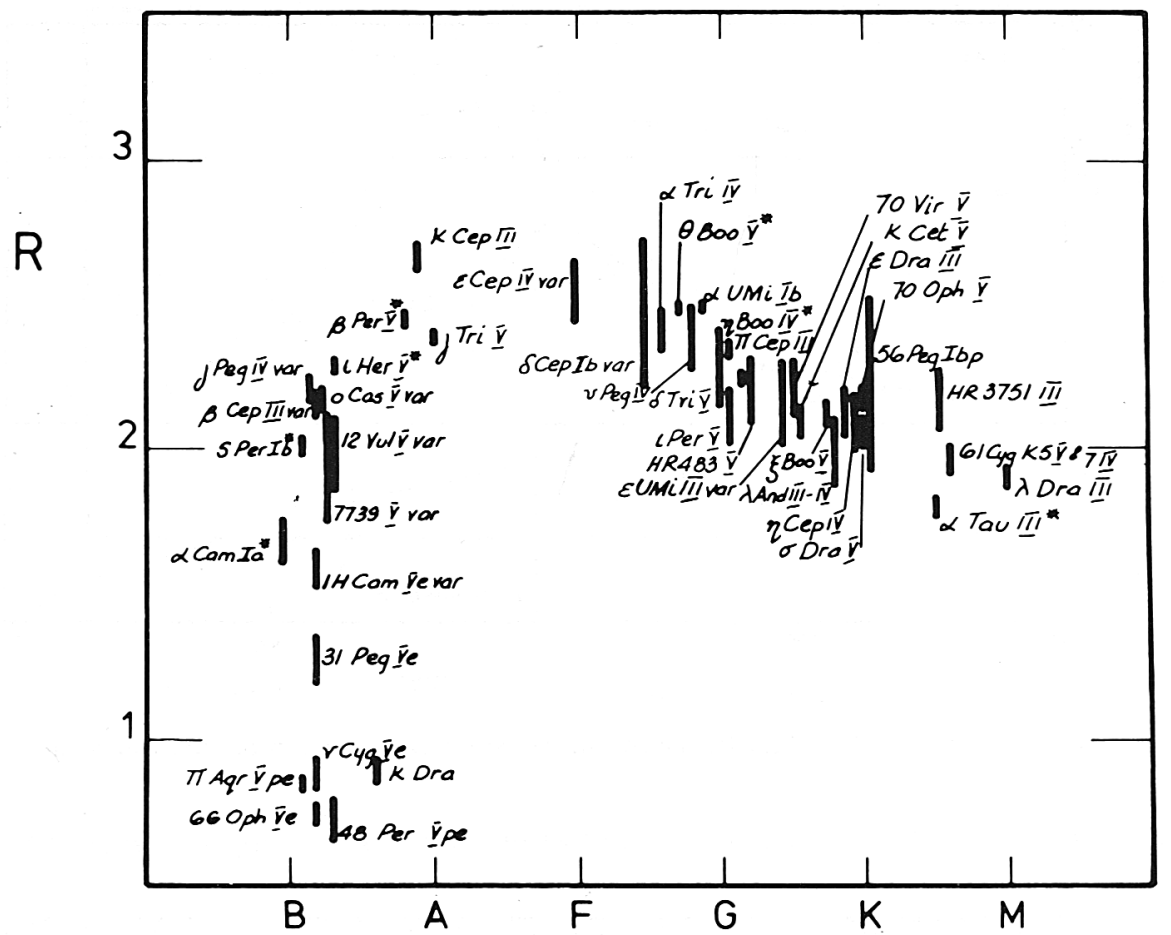

Fig. 1. Ranges of $R$ for all the observed stars plotted against spectral type.

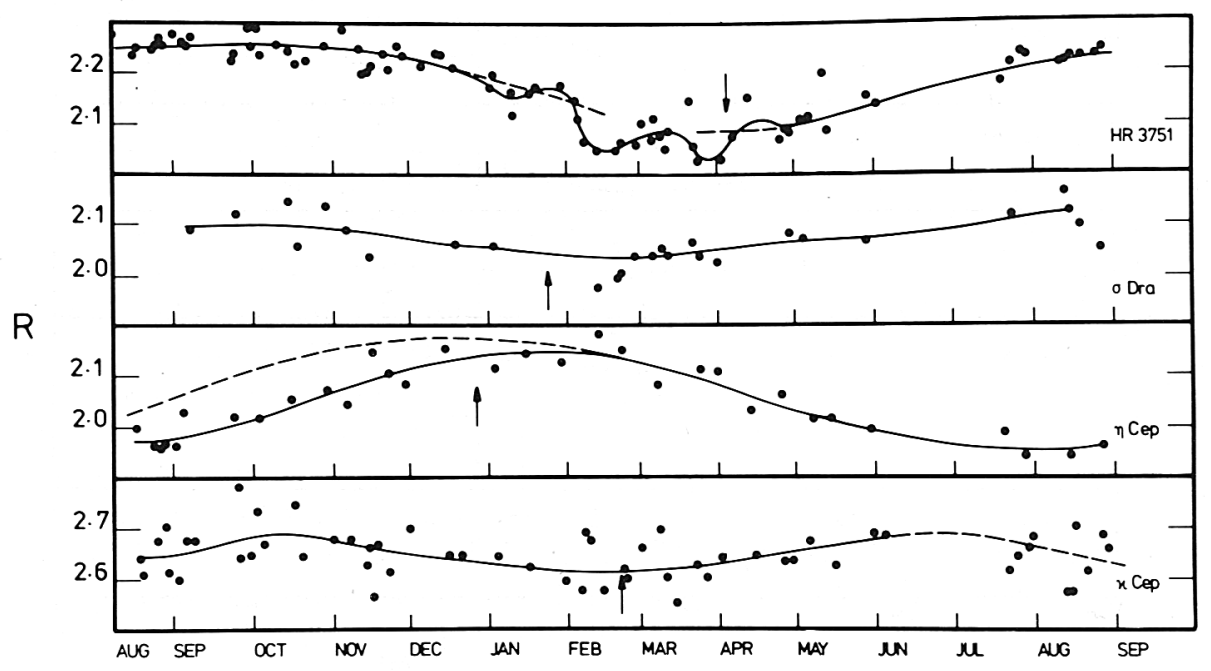

Fig. 2. Values of $R$ for the four stars HR 3751, $\sigma$ Dra, $\eta$ Cep, and $\kappa$ Cep plotted against date. 


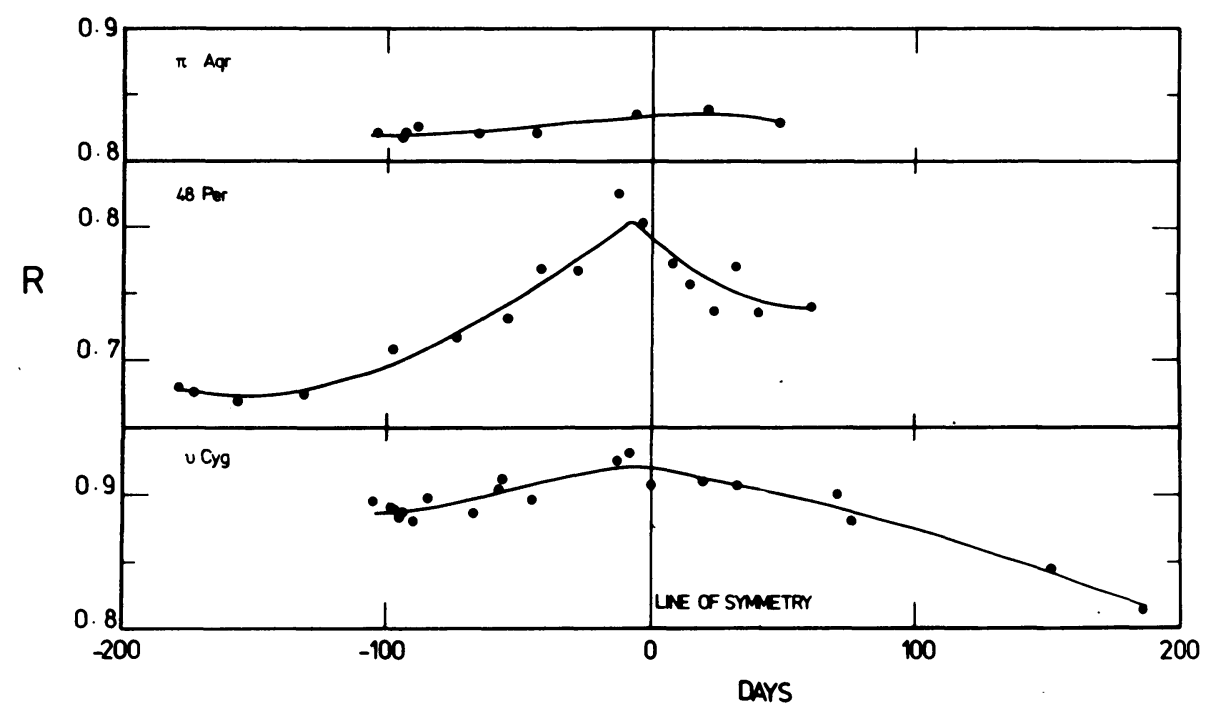

Fig. 3 Values of $R$ for three Be stars having $\mathrm{H} \alpha$ strongly in emission, plotted against time.

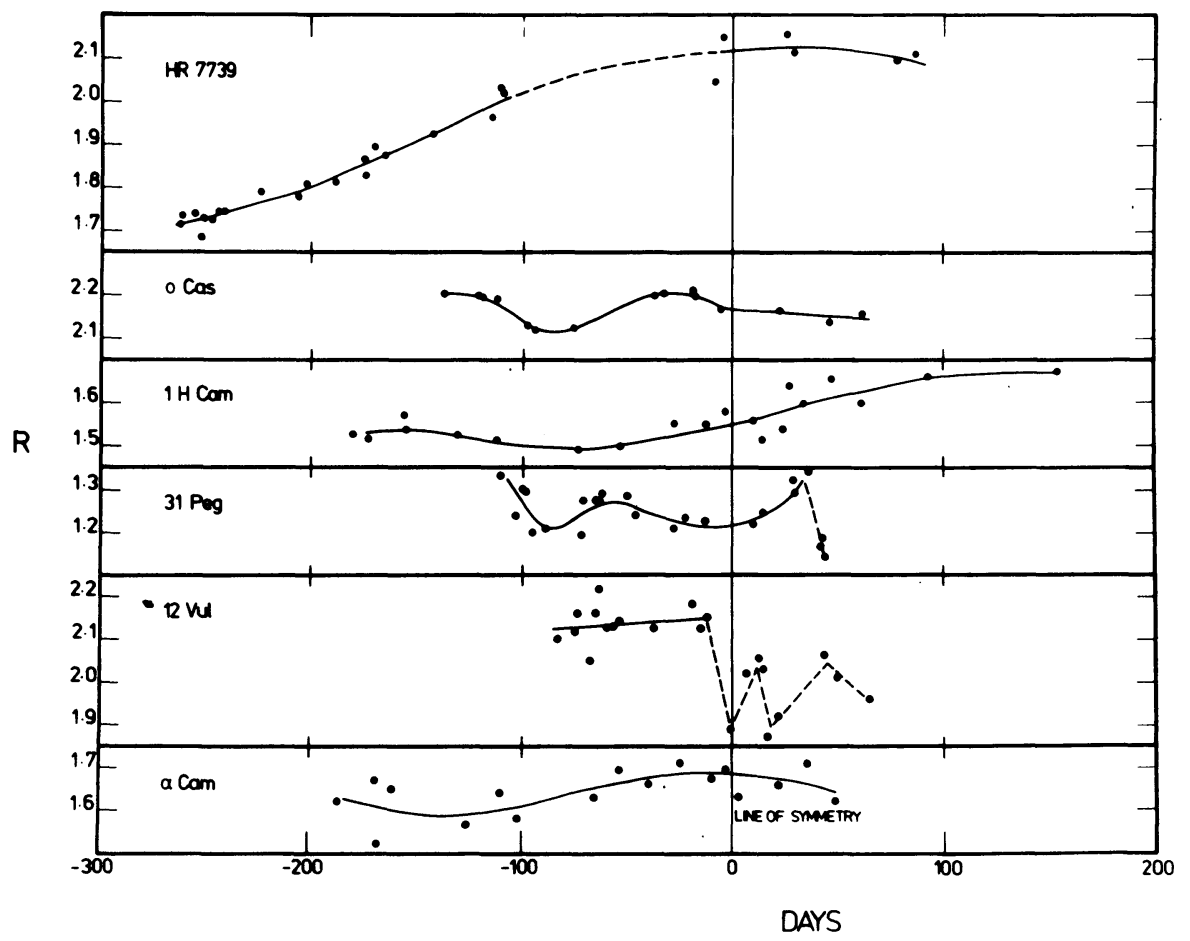

Fig. 4. Values of $R$ for six Be stars plotted against time. 


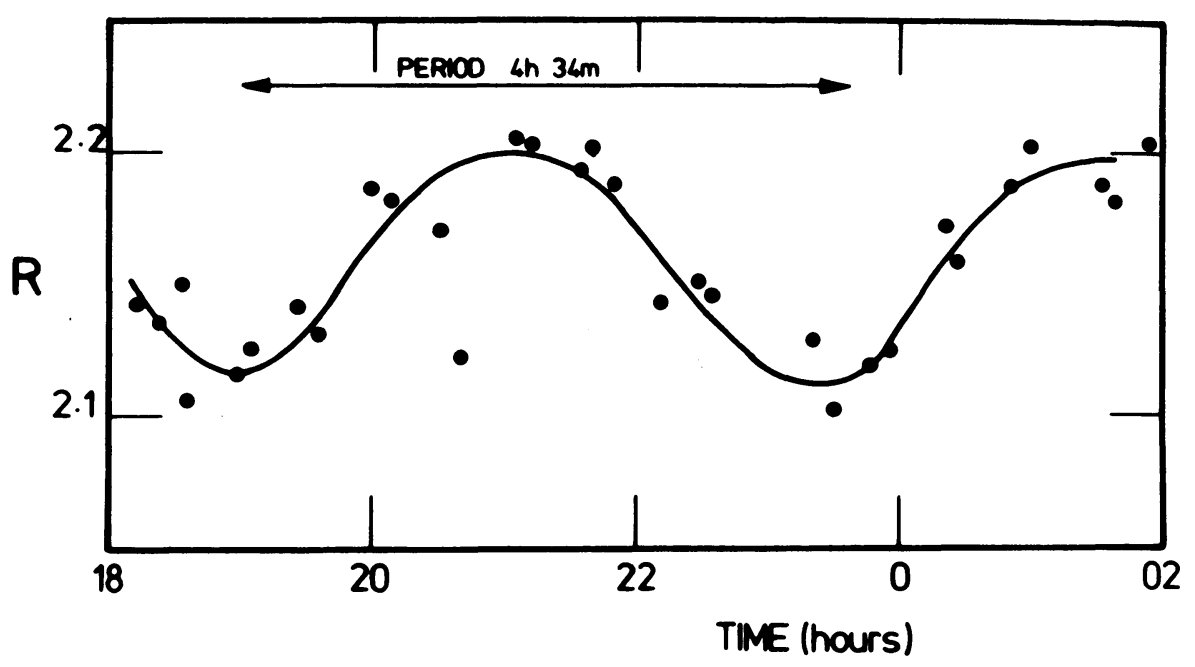

Fig. 5. Values of $R$ for $\beta$ Cep plotted during eight hours of the night 13-14 November 1972.

\section{Effect of Doppler Shift, Particularly that Due to the Earth's Annual Motion}

A narrow-band filter, if set near to the centre of an emission or absorption line, is evidently a sensitive device for the detection of any change in wavelength due, for example, to the Doppler effect.

There is good, but not certain, reason to believe that the Doppler effect consequent upon the pulsation of $\beta$ Cep causes the curve of Figure 5.

Another such variation will come from the Earth's Annual Motion around the Sun which produces an apparent sinusoidal change in each star's radial velocity of amplitude approximately $30 \cos$ (ecliptic latitude) $\mathrm{km} \mathrm{s}^{-1}$, equivalent to $0.66 \cos$ (ecliptic latitude) $\AA$ at $\mathrm{H} \alpha$, with a period of one year. This brings about relative movement of stellar spectrum and filter passband which is perhaps best seen as the filter profile scanning across the $\mathrm{H} \alpha$ profile. Assume that the $\mathrm{H} \alpha$ line is symmetrical and in absorption. Consider first the case when the filter passband is exactly centred on the laboratory value of the wavelength of $H \alpha(6562.8 \AA)$ and the star has zero radial velocity. Starting from the moment of maximum red shift of the spectrum, the filter profile starts from rest, crosses the centre of the $\mathrm{H} \alpha$ line and stops again after 6 months: it then returns across it during the next 6 months. This will lead to a plot of $R$ with time showing two equally spaced humps and two troughs -i.e. to a period of 6 months.

If, on the other hand, the filter passband is appreciably offset from $6562.8 \AA$, it will not cross the centre of the $\mathrm{H} \alpha$ profile at all during one year, only encroaching into one wing of $\mathrm{H} \alpha$, and so the observed variation in $r$ will show only one hump and one trough in a year, i.e. a period of 12 months.

If we consider cases with the offset decreasing toward zero, the peak of the 12 monthly curve first becomes double, then the separation of the twin peaks increases 
to the point that, when the offset is zero, the separation is 6 months and we have reached the case of 6 month periodicity first considered above.

Among the stars that were observed over an adequate period were cases of wide and narrow $\mathrm{H} \alpha$ profile, positive and negative stellar radial velocity, as well as $\mathrm{H} \alpha$ in emission and absorption.

Provided there are no large intrinsic stellar variations, the above effect is clear and rational. A careful examination of all the observations shows, in fact, that the filter passband was offset by $0.6 \AA$, rather more than was anticipated. Once this figure was available, crude measures of the radial velocities of individual stars could also be deduced.

\section{Intrinsic Stellar Variations}

The reduced data of 14 stars are to be seen in Figures 2, 3 and 4. Each plotted point is the mean of all data observed during the night - usually 4 independent measures. In Figure 2, the symmetry points of the variation due to the Earth's Annual Motion are marked with arrows at the appropriate dates. In Figures 3 and 4, the curves have been moved sideways (and the actual dates thereby lost) so that symmetry of all will be about the same vertical line marked 'line of symmetry'.

Figure 2 shows the data for four stars with $\mathrm{H} \alpha$ in absorption - three of spectral type $K$ and one of type B. They include HR3751 and $\kappa$ Cep, the two circumpolar stars chosen for intensive observation. $\kappa$ Cep shows no indication of intrinsic variation but its curve is of interest because it shows one of the intermediate cases due to the Earth's motion, mentioned earlier. The main minimum is in late February and there are two maxima, one clearly evident in the first week of October and the second, far less so, in June.

HR3751 shows an unexceptionable 12 month variation but, incidentally, does just seem to show 3 periods of about $\mathbf{4 0}$ days which could be the hoped for evidence of rotation mentioned in (b) in the Introduction.

$\sigma$ Dra has a high ecliptic latitude $\left(81^{\circ}\right)$ and little effect of the Earth's motion is to be expected, nor is seen. There is slight evidence for intrinsic changes in $\mathrm{H} \alpha$. The fourth star, $\eta$ Cep, appears at first sight to show the correct response to the Earth's motion and to exhibit no stellar variation. However, the maximum does not occur at the correct time (shown by the arrow). To bring the curve to a position such as the dashed line, requires some stellar change and/or considerable asymmetry in the $\mathrm{H} \alpha$ profile.

Figure 3 shows three stars with $\mathrm{H} \alpha$ in emission. The curves have been moved sideways so that one or other of the symmetry points for the Earth's motion coincide in time with the vertical line. It can be seen that the general shape can be explained by Earth's motion. For $v$ Cyg, only the last two points show any divergence from a perfectly smooth curve, and for those the observations were made when the star was low in a bright sky. The individual points of 48 Per do show rather more variation about the smooth curve than is to be expected. The curve of the third star, $\pi$ Aqr has the general shape of the other two stars.

Of the stars of Figure 4 (presented in the same way as those of Figure 3), three are classed as e-type, two others have high values of $v \sin i$, and one is of spectral type O9.5Ia, and all show some degree of emission. 
It will be seen that except for HR7739, intrinsic stellar variations swamp any changes due to the Earth's motion. Judged by the degree of internal consistency of the individual readings (during one night, and from night to night and also from star to star) there is real variation with a time scale of the order of one day for 1HCam between +10 and +40 days, 12 Vul between 0 and +50 days, and for $\alpha$ Cam throughout the period of the observations. No deductions can be made about shorter period variability.

\section{The Practical Use of a Narrow-Band Interference Filter}

Heating a filter of this sort increases the passband wavelength, tilting decreases it and, as a corollary of the latter, decreasing the f-number of the beam through the filter likewise decreases the passband wavelength and somewhat alters its shape.

It is usual to specify a filter having a central value positioned a small amount longwards of the desired wavelength. The amount can, to a certain extent, be calculated, but it is essential to confirm, in practice, that the final setting is correct. This needs some, or all, of the adjuncts of a photometric laboratory.

In the present instance, two methods were used:

(a) the thermostatted filter was placed in front of the slit of a solar spectrograph. The photographed spectra at different filter tilts confirmed that the chosen tilt gave the correct passband;

(b) the complete photometer was fed with light from a low pressure hydrogen lamp, and readings were taken for different tilts.

As stated, it was felt that the tilt setting gave the correct passband to an accuracy better than $0.5 \AA$ whereas the stellar observations showed it to have an error of $0.6 \AA$. Halfway though the observing season the filter was checked and did not seem to have changed in any way. The work had begun in August 1972. By August 1973, readings on a number of stars which did not seem to show any intrinsic variation, were systematically different from what they had been a year earlier. The difference was small and considered acceptable. However, during September and October this difference rapidly increased and in November it was assumed that one of the optical components was changing and the observations were discontinued.

On repeating the checks there was no convincing evidence that the passband had changed although there did appear to be more variation across the narrow-band filter (corresponding to slight visible patterning) than had previously been noticed. The cause could have been due to a change in either of the filters (in shape of passband or in general loss of transparency) or to a change in the grey filter; it was not possible to determine which.

\section{Conclusions}

(1) A 12-in. telescope, a $3.5 \AA \mathrm{H} \alpha$ filter and a photon counting photometer are together satisfactory for monitoring variations in stars brighter than the fifth magnitude: such as instrument can be dedicated to one programme for a long period.

(2) It is important to have access to a photometric laboratory for setting the filter 
equally important to check both filter passbands and their through-put accurately at regular intervals.

(3) The method is a sensitive one for detecting change in the radiation passing through the $\mathrm{H} \alpha$ filter but it does not allow one to differentiate between changes in the $\mathrm{H} \alpha$ profile and wavelength changes in the spectrum due to the Doppler shift.

(4) Of the Be stars observed, three, although in strong emission, showed negligible variation while six others showed appreciable changes with a timescale of the order of one day.

(5) The instrument is particularly suitable for observing short period changes: as no comparison star is used, very efficient use can be made of the observing time. It is, in fact, intended to search with it for rapid changes $(1 \mathrm{~min}$ to $1 \mathrm{~h})$ in a few Be stars.

\section{DISCUSSION}

Bidelman: Wouldn't it be worthwhile to use a somewhat wider filter? In many stars the emission is appreciably wider than 3.5 ångström.

Butler: I chose the width to deal with late types, solar types, where it very roughly corresponds to the pass band of the profile of $\mathrm{H} \alpha$ in the sun. For the emission lines, of course, it is rather nice to have a narrow filter. Also, the wider the filter, the more the noise creeps in.

McLean: At the University of Glasgow, Scotland, we have developed two instruments employing narrow band $\mathrm{H} \alpha$ and $\mathrm{H} \beta$ interference filters, viz., a line profile scanning photometer and a line profile scanning polarimeter. Line profile scans are obtained by controlled tilting of the interference filters in collimated light beams. Excellent stability and accuracy in recording line profiles is obtained with the photometer (developed mainly by T. H. A. Wyllie), in which the filters are housed in a thermostatted enclosure. This instrument also employs continuous guidance on the star image and a fixed monitor channel which is ratioed with the scanning channel. Small and rapid changes in intensity and/or wavelength shifts are easily identified. Only for $\gamma$ Cas has variability been observed at $\mathbf{H} \beta$ on a time scale of minutes (Clarke, D., McLean, I. S., and Wyllie, T. H. A.: 1975, Astron. Astrophys., in press).

Harmanec: It would be useful to make $\mathrm{H} \alpha$ and $\mathrm{H} \beta$ measurements simultaneously.

Butler: I agree. I had toyed with the idea of using a dichroic filter for this purpose. 